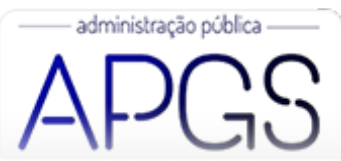

Administração Pública e Gestão Social ISSN: 2175-5787

apgs@ufv.br

Universidade Federal de Viçosa

Brasil

\title{
Comprometimento Institucional: um modelo interpretativo para o serviço público brasileiro
}

\author{
Terra de Andrade, Diego César \\ Comprometimento Institucional: um modelo interpretativo para o serviço público brasileiro \\ Administração Pública e Gestão Social, vol. 13, núm. 2, 2021 \\ Universidade Federal de Viçosa, Brasil \\ Disponível em: http://www.redalyc.org/articulo.oa?id=351566014010
}

\section{(c) (1) $\$(\Theta$}

Este trabalho está sob uma Licença Creative Commons Atribuição-NãoComercial-NãoDerivados 3.0 Internacional 


\title{
Comprometimento Institucional: um modelo interpretativo para o serviço público brasileiro
}

\author{
Institutional commitment: an interpretive model for the Brazilian public service \\ Compromiso institucional: un modelo interpretativo para el servicio público brasileño
}

\author{
Diego César Terra de Andrade \\ Universidade Nove de Julho, Brasil \\ diego.terra@ifsuldeminas.edu.br
}

Redalyc: http://www.redalyc.org/articulo.oa?

$\mathrm{id}=351566014010$

Recepção: 15 Junho 2020

Aprovação: 11 Setembro 2020

Publicado: 01 Abril 2021

\section{Resumo:}

Objetivo da pesquisa: As peculiaridades encontradas no setor público brasileiro demandam pesquisas direcionadas ao entendimento dos fenômenos do comportamento organizacional. Destarte, o objetivo central desta pesquisa é avaliar um modelo interpretativo do comprometimento institucional que melhor explique o comportamento dos servidores públicos.

Enquadramento teórico: A revisão bibliográfica engloba os construtos Engajamento no Trabalho, Satisfação no Trabalho e Articulação de Valores Pessoais e Valores Organizacionais.

Metodologia: $\mathrm{O}$ modelo foi testado em amostra composta de $\mathrm{n}=902$, por meio de um survey on-line, disposta em escala do tipo Likert. Os dados foram analisados utilizando a técnica de modelagem de equações estruturais (MME).

Resultados: As três hipóteses do modelo inicial, bem como a equação do modelo integrativo, foram confirmadas. O modelo estrutural que trouxe o melhor ajuste foi o terceiro ( $\mathrm{R} .=47,5 \%$ e Q2 $=0,207)$.

Contribuições teóricas e práticas: Como contribuição teórica o trabalho apresenta, ainda, que o construto Articulação dos Valores Organizacionais e Valores Pessoais exerce papel mediador e o construto Engajamento no Trabalho é um antecedente.

Palavras-Chave: Comprometimento Institucional, Setor Público, Modelo Interpretativo, Modelagem de Equações Estruturais.

\section{Abstract:}

Research objective: The peculiarities found in the Brazilian public sector demand research aimed for understanding the phenomenon of organizational behavior. Thus, the main objective of this research is to evaluate an interpretative model of institutional commitment, which better explains the behavior of public servants.

Theoretical framework: The bibliographic review includes the constructs Engagement at Work, Job Satisfaction and Articulation of Personal Values and Organizational Values.

Methodology: The model was tested on a sample composed of $\mathrm{n}=902$, through an online survey, arranged on a Likert scale. The data were analyzed using the structural equation modeling (MME) technique.

Results: The three hypotheses of the initial model, as well as the equation of the integrative model, were confirmed. The structural model that brought the best fit was the third $(\mathrm{R} 2=47.5 \%$ and $\mathrm{Q} 2=0.207)$.

Theoretical and practical contributions: As a theoretical contribution, the work also presents that the construct Articulation of Organizational Values and Personal Values plays a mediating role and the construct Engagement at Work is an antecedent.

KEYWORDS: Institutional commitment, Public sector, Interpretive Model, Structural Equation Modeling.

\section{Resumen:}

Objetivo de la investigación: Las peculiaridades encontradas en el sector público brasileño exigen una investigación orientada a comprender los fenómenos del comportamiento organizacional. Así, el principal objetivo de esta investigación es evaluar un modelo interpretativo de compromiso institucional que explique mejor el comportamiento de los servidores públicos.

Marco teórico: La revisión bibliográfica incluye los constructos Compromiso en el trabajo, Satisfacción laboral y Articulación de valores personales y valores organizacionales.

Metodología: El modelo se probó en una muestra compuesta por $\mathrm{n}=902$, mediante una encuesta en línea, ordenada en escala Likert. Los datos fueran analizados mediante la técnica de modelado de ecuaciones estructurales (MME). 
Resultados: Se confirmaron las tres hipótesis del modelo inicial, así como la ecuación del modelo integrador. El modelo estructural que mejor calzó fue el tercero ( $22=47,5 \%$ y Q2 $=0,207)$.

Aportes teóricos y prácticos: Como aporte teórico, el trabajo también presenta que el constructo Articulación de Valores Organizacionales y Valores Personales tiene un papel mediador y el constructo Engagement at Work es un antecedente.

Palabras Clave: compromiso institucional, Sector público, Modelo interpretativo, Modelos de ecuaciones estructurales.

\section{INTRODUÇÃO}

Sob uma ótica marxista, a busca pelo trabalhador comprometido e produtivo, pode ser interpretada como um sonho impossível frente ao inevitável conflito entre capital e trabalho. Numa perspectiva das relações industriais tradicionais, pode ser considerada como simplista e ingênua, nos termos da qual os resultados são necessariamente um produto de uma pechincha e um acordo (Cunha, Rego, Cunha, \& Cabral-Cardoso, 2016). Todavia, numa perspectiva psicológica, o andamento do trabalhador comprometido e produtivo temse apresentado como um objetivo de mérito, ainda que complexo - "um objetivo que pode ser logrado se incrementado o conhecimento acerca das atitudes e comportamentos" (Staw, 1986, p. 40).

O comprometimento com o trabalho, designação que aqui se utiliza em detrimento da concepção mais restrita de comprometimento com a função, de acordo com Cunha, Rego, Cunha e Cabral-Cardoso (2016), é um dos construtos mais estudados no âmbito do Comportamento Organizacional. De acordo com Jayaratne (1993), o comprometimento ocupa um lugar central nas investigações desde o livro Job Satisfaction, de 1935, de Robert Hoppock.

Embora seja tentadora e até mesmo intuitiva a ideia de que o trabalhador mais comprometido é o mais produtivo, a definição de comprometimento está normalmente mais próxima dos estados afetivos e das cognições que lhes são associadas do que a questão da produtividade. A razão disso deve-se ao fato de que só a partir da inclusão de elementos cognitivos é que se pode obter uma plataforma conceitual para o relacionamento com a variável desempenho.

Nesse sentido, cabe observar que o Comprometimento Organizacional pode resultar de três fatores: o indivíduo, o trabalho e a interação indivíduo-trabalho. Assim, o objetivo central desta pesquisa é avaliar um modelo interpretativo, a partir dos três fatores anteriormente elencados, do comprometimento institucional que melhor explique o comportamento do servidor público brasileiro.

$\mathrm{Na}$ busca desse objetivo três construtos serão utilizados: Articulação entre Valores Pessoais e Valores Organizacionais, como medida da interação indivíduo-trabalho; Satisfação no Trabalho, como medida do trabalho; e Engajamento no Trabalho, como medida do indivíduo. No desenvolvimento do texto será explicitada a razão dessas escolhas.

\section{Engajamento no Trabalho, Satisfação no Trabalho e Articulação de Valores Pessoais e Valores Organizacionais}

O construto multifatorial Engajamento no Trabalho, de acordo com Schaufeli, Salanova, González-Romá e Bakker (2002, p. 74), é "um estado mental positivo de realização relacionado ao trabalho e que se caracteriza por vigor, dedicação e absorção, independente do local ou atividade que se desempenha”. Nesse sentido, vigor constitui-se de altos níveis de energia mental durante o trabalho, investimentos de esforços durante a realização de tarefas e persistência para ultrapassar dificuldades nesse contexto. Dedicação, explicam os autores, "compreende um estado de alto grau de envolvimento com uma atividade, durante a qual o indivíduo experimenta prazer, inspiração, entusiasmo e reconhece significado no trabalho”. Por sua vez, a absorção refere-se a um alto nível de concentração no trabalho, durante o qual o indivíduo nem percebe o tempo passar e praticamente não vê distinção entre si e as tarefas que realiza. 
Para Bakker, Schaufeli, Leiter e Taris (2008), os trabalhadores engajados são aqueles que possuem altos níveis de emoções positivas, melhor saúde física e psicológica, criam seus próprios recursos, trabalham duro (vigor), são imersos em suas atividades (dedicação) e se sentem de forma plena e altamente concentrados em seus trabalhos (absorção).

De acordo com Meleiro e Siqueira (2005), Padovam (2005), Siqueira (2003; 2005) e Tamayo (1998), a satisfação no trabalho seria um sentimento altamente compassivo às políticas e condutas gerenciais, sobretudo aquelas que determinam os procedimentos e a divisão de retornos (percepções de justiça no trabalho) para os comportamentos de trabalhadores, que indicam se a empresa está\# comprometida com os seus colaboradores, ou seja, o quanto a empresa se preocupa com o bem-estar deles (percepçóes de suporte organizacional) e o quanto ela (a empresa), estaria disposta a compensar seus empregados pelos esforços e todos os investimentos que eles aplicam na organização (reciprocidade organizacional).

De acordo com Wright e Davis (2003), o ambiente laboral é constituído por dois componentes: características do trabalho e contexto do trabalho. As características do trabalho são os aspectos do trabalho de uma pessoa ou responsabilidades do cargo que contribuem para o seu estado psicológico, como a significância do trabalho, que afeta o desenvolvimento, crescimento e disposição dos indivíduos para o trabalho. Já as variáveis do contexto do trabalho são formadas por características organizacionais como sistemas, metas e graus de formalização. Ademais, nas variáveis do contexto do trabalho espera-se que os indivíduos desenvolvam seus deveres.

Consonante com essas ideias, a presente pesquisa empregou o entendimento de que a Satisfação no Trabalho é um atributo afetivo, referente ao nível de contentamento do indivíduo frente a sua atividade laboral, sendo, portanto, "uma variável atitudinal com enfoque pós-cognitivo e caráter multidimensional” (Traldi \& Demo, 2012, p. 300). Dentro desse escopo, a satisfação é "um elo afetivo com o trabalho, que se desenvolve a partir de cognições elaboradas pelo empregado sobre sua relação de troca social com o sistema" (Siqueira, 1995, p. 160).

De acordo com Kluckhohn e Strodtbeck (1961, p.439), "um valor é uma concepção, explícita ou implícita, própria de um indivíduo, ou característica de um grupo, acerca do desejável, que influi na seleção de modos, meios e finalidades de ações acessíveis". Rokeach (1973, p. 5) define valor como uma "crença duradoura de um modo específico de conduta ou estado final de existência individual ou socialmente preferível em oposição a outro". Já para Schwartz (1994), valores são metas transituacionais desejáveis, que variam em importância e servem como princípios guias na vida de uma pessoa ou entidade social.

Para Oliveira e Tamayo (2004), os indivíduos, ao iniciarem nas organizações, carregam os seus valores pessoais adquiridos no meio social a que pertencem. Em relação aos valores organizacionais, as organizaçóes, desde seu nascimento, constroem um sistema de valores próprios, que aparecem em função de problemas característicos da sua própria razão de existir. Sendo assim, Canova e Porto (2010) consideram que a existência desses valores organizacionais antecede o ingresso das pessoas na organização.

Existem alguns modelos que buscam compreender a articulação entre os valores pessoais e os organizacionais. Por exemplo, o modelo de Nelson (2011) postula que os valores organizacionais influenciam os valores pessoais, bem como os valores pessoais influenciam os valores organizacionais. Trata-se de dois conjuntos diferentes de valores, mas que possuem as mesmas dimensões, que expressam e representam motivações semelhantes do indivíduo e do grupo, do trabalhador e da organização.

Sob este prisma, os valores são representações cognitivas, socialmente compartilhadas, de necessidades individuais, no caso dos valores pessoais, e de exigências e objetivos grupais, no caso dos valores organizacionais. As particularidades gerais dos valores são comuns a ambos os sistemas. Ou seja, reconhece que o isomorfismo motivacional consiste na correspondência biunívoca dos tipos motivacionais de valores do trabalhador (pessoais), e dos valores da organização (organizacionais).

Nesse tipo de abordagem, a relação entre os tipos motivacionais de valores do trabalhador, com os conjuntos de valores da organização, implica a correspondência de metas entre o trabalhador e a organização. 
A título de exemplo, o trabalhador conta entre suas metas e motivações cotidianas a procura de sucesso, de prestígio e de respeito da tradição. A organização também procura sucesso, prestígio e respeitar a tradição. $\mathrm{O}$ que se verifica nesse caso é a correspondência de metas do empregado e da coletividade organizacional. Ou seja, há um ajuste $(f i t)$ entre os valores de ambos.

\section{Comprometimento Organizacional, Hipóteses e Modelos}

O Comprometimento Organizacional é um construto que desde algum tempo e abundantemente tem provocado grande interesse entre os investigadores das organizações (Allen \& Mayer, 1996; Jaramilo, Mulki, \& Marshall, 2005; Meyer, Stanley, Hercovitch, \& Topolnytsky, 2002; Morrow, 2011). Uma das razões que pode explicar esse interesse advém de o comprometimento de se relacionar positivamente ao desempenho, conforme demonstrado em estudo meta-analítico de Riketta (2005).

Em outro trabalho meta-analítico, Cooper-Hakim e Viswesvaran (2005), ao analisarem as relações entre os múltiplos comprometimentos organizacionais, assinalam a redundância conceitual e o caráter multidimensional do construto.

Nesse sentido, o conceito adotado neste trabalho é o de Brief(1998), para quem o comprometimento com a organização advém de "um estado emocional expresso através da avaliação afetiva e (ou) cognitiva de uma experiência de trabalho, com algum grau de favorabilidade ou desfavorabilidade” (p. 86).

Já sobre seu caráter multidimensional, uma grande diversidade de variáveis tem vindo a ser usadas para explicar o Comprometimento Organizacional. Os modelos resultantes, todavia, de acordo com Cunha et al. (2016), podem ser agrupados em três grandes tipos:

1 - As políticas e práticas organizacionais, como, por exemplo, a política salarial, os estilos de chefia, o processo decisório, promoções, etc.;

2 - As características da função, como, por exemplo, a variedade, identidade, significado, autonomia e feedback das tarefas;

3 - As características individuais, tais como a autoestima, o locus de controle, a necessidade, o engajamento, motivação pelo sucesso, etc.

Por conseguinte, o histórico dos estudos sobre o Comprometimento Organizacional tem sido agrupado também em três grandes modelos de investigação:

1 - Os modelos centrados no indivíduo, que são aqueles que procuram identificar as influências disposicionais mais ligadas ao comprometimento. A questão subjacente é: quais as características individuais que tornam determinados indivíduos "naturalmente" mais ou menos comprometidos com a organização?

2 - Os modelos centrados nas situações analisam as relações entre o contexto ou ambiente de trabalho e os níveis de comprometimento. A questão implícita é: quais as características do contexto de trabalho que induzem a maior ou menor comprometimento com a organização?

3 - Os modelos centrados nas interações investigam o modo como o comprometimento pode ser explicado pelo grau de ajustamento entre as características individuais e as características encontradas na organização. A questão oculta é: como as características individuais e as características organizacionais se combinam para gerar o comprometimento organizacional?

Parece ser simplista, e porque não ingênuo, tratar uma dimensão tão complexa de ser medida frente a determinar se as características individuais surgem como determinantes principais do comprometimento organizacional, se esse é um papel desempenhado pelas características do trabalho ou da congruência (ou identificação) entre valores pessoais e organizacionais. Neste sentido, este trabalho propõe um avanço na forma de compreender o fenômeno do Comprometimento Organizacional através de um modelo integrativo dos três grandes modelos até então propostos pela literatura.

Além do avanço de ser um modelo integrativo, serão utilizadas medidas já testadas e adaptadas ao serviço público brasileiro, pois o que se busca é seguir a indicação de Andrade e Ramos (2018), que afirmam 
a necessidade de se testar e adaptar unidades de medidas direcionadas ao tão específico comportamento organizacional, observado nas instituições públicas nacionais. Assim, ao invés de tratar o construto como Comprometimento Organizacional, será utilizado o termo Comprometimento Institucional.

Assim, o modelo integrativo aqui sugerido será facultado pela seguinte equação:

$\mathrm{CI}=f(\mathrm{P} \times \mathrm{T} \times \mathrm{APT} \times \mathrm{E})(1)$

Onde:

CI representa o comprometimento institucional;

P representa a personalidade ou quaisquer outras disposições individuais;

T representa como o indivíduo vê os multifatores inerentes ao trabalho; APT a relação indivíduo-trabalho;

E representa o erro, que é inerente a todas as intenções de generalizações comportamentais, seja em pesquisas quantitativas, seja em pesquisas qualitativas.

Se analisada a fundo a proposta desta equação, nota-se dois elementos principais: a personalidade e o trabalho. Isso corrobora com a proposta de Mowday, Porter e Steers (1982), que afirmam que para a definição do construto deve ser observado:

1 - A forte crença e aceitação dos valores da organização;

2 - O forte desejo de manter o vínculo com a organização;

3 - A intenção de se esforçar em prol da organização.

O que enseja as seguintes hipóteses de pesquisa:

H1 - O grau de ajustamento entre as características do indivíduo e as da organização (que aqui será medido através de uma escala que analisa a congruência entre os Valores Pessoais e os Valores Organizacionais) influencia positivamente a satisfação no trabalho.

Segundo Siqueira (2008), a satisfação no trabalho é "um dos múltiplos conceitos que abordam a afetividade no ambiente de trabalho, ou, mais especificamente, como um vínculo afetivo do indivíduo com o seu trabalho" (p. 266). Ainda para a mesma autora, essa passa a ser compreendida como um resultado (output) do ambiente organizacional.

H2 - As características encontradas no trabalho (que aqui será medido através de uma escala de Satisfação no Trabalho) influenciam positivamente o engajamento no trabalho.

A afetividade, de acordo com Siqueira (2008), é um dos três componentes psicossociais do conceito de bem-estar no trabalho, ao lado de envolvimento no trabalho e comprometimento organizacional afetivo. Ambos são construtos que mantêm forte semelhança com o engajamento no trabalho.

H3 - Independente das características encontradas no local de trabalho, o grau de ajustamento entre as características do indivíduo e as da organização influenciam positivamente os níveis de comprometimento superiores com a instituição (que aqui será medido através de uma escala de Engajamento no Trabalho).

Vroom (1966) afirma que os indivíduos sentem-se atraídos por uma organização na expectativa de que seus valores pessoais coadunem com os valores emanados pela organização. Todavia, o indivíduo poderá descobrir, após algum tempo na organização, que não se encaixa nela e, consequentemente, experimentar o que Schneider (1987) e Schneider, Goldstein e Smith (1995) denominam atrito (attrition). Esse desacordo pode resultar na falta de engajamento no trabalho. Portanto, o oposto pode ser verdadeiro, o ajuste entre os valores pessoais e organizacionais podem aumentar o engajamento no trabalho.

Assim, a Figura 1 esquematiza as hipóteses passíveis de verificação empírica, por meio da proposição dos três modelos teóricos a serem testados na busca do que melhor responde ao objetivo deste trabalho. 




Figura 1: Modelos testados no estudo

Fonte: elaborada pelos autores.

\section{Procedimentos Metodológicos}

O quadro de referência foi influenciado pelo Positivismo, conforme a classificação de Brujne, Herman e Schoutheete (1977). Quanto à tipologia, segundo Burrel e Morgan (1982), o trabalho é essencialmente funcionalista. Já quanto à tipologia preconizada por Babbie (1996), este estudo encontra amparo no paradigma Estruturo-Funcionalista. O modelo teórico adota um sistema dinâmico e as inter-relações entre as variáveis se constituirão em funções dinâmicas, possuindo caráter exploratório causal (Aaker, Kumar, \& Day, 2001; Hair, Anderson, Tatham, \& Black, 2005; Malhotra, 2006) e de avaliação quantitativa (Creswell, 2014).

A unidade de análise foram instituições públicas do Brasil. A população foi compreendida por servidores públicos da ativa das diferentes esferas: federal, estadual e municipal. As mais diferentes funções, indistintamente, participaram da pesquisa. Portanto, a amostra do estudo caracteriza-se como não probabilística por conveniência. Referente à variável sexo, $52 \%$ dos respondentes são do sexo masculino e $48 \%$, do sexo feminino. $71,27 \%$ da amostra tem menos de 10 anos de exercício na instituição. Com relação ao nível de escolaridade, $1,55 \%$ possui ensino fundamental, $16,41 \%$ possui ensino médio, $22,39 \%$ possui o ensino superior e $59,65 \%$ possui pós-graduação.

A amostra, que é do tipo conveniência, foi composta de 906 respondentes, sendo excluídos 4 outliers. Levando-se em consideração o indicado por Hair, Anderson, Tatham e Black (2005), deve-se obter pelo menos cinco respondentes para cada item independente analisado. Assim, esse critério amostral foi atendido em sua totalidade, uma vez que a soma dos itens independentes das três escalas que foram utilizados é da ordem de 40 itens.

Como dito, o instrumento desta pesquisa foi constituído de três escalas. Entretanto, inicialmente os respondentes marcaram um visto no termo de consentimento da pesquisa. Em seguida, utilizaram os instrumentos padronizados para fornecer informações sobre dados sociodemográficos, que além das questões tradicionais - como, por exemplo, cargo, ano de admissão e sexo - apresentou perguntas mais específicas para levantar se já exerceu algum cargo de gestão anteriormente a sua entrada no serviço público e no próprio serviço público, entre outras. Essas questões poderão auxiliar na compreensão do microcomportamento organizacional por meio de seus indivíduos e abrir possibilidades de novos trabalhos, que no momento fogem do escopo e objetivos dessa pesquisa.

Quanto aos instrumentos para aferir os valores pessoais e valores organizacionais, cogitou-se a utilização dos trabalhos de Oliveira e Tamayo (2004) e Schwartz (2005), respectivamente. Segundo meta-análise realizada por Torres, Porto, Vargas e Fischer (2015) e a pesquisa de Tamayo (2007), esses trabalhos são os mais utilizados nessa temática em publicações brasileiras. No entanto, optou-se pelo uso da escala Articulación entre la Persona y la organización (APO), elaborada por Ramos, Jordão e Morais (2013), por tratar-se de um instrumento que contempla os dois construtos em conjunto, e são elaborados pelos mesmos autores, evitando assim divergências epistemológicas. $\mathrm{O}$ instrumento original era composto por 19 variáveis que, após sua validação fatorial, passou a contar com 16 questões. Cabe ressaltar que o instrumento já foi utilizado no serviço público brasileiro por Andrade (2019), e mostrou-se estatisticamente fiável. Também foi levado 
em consideração - neste e nos demais instrumentos - que quanto menor fosse o questionário final, maior poderia ser a taxa de respostas.

O APO possui três fatores: fit (ou ajuste), com seis itens, misfit (ou conflito), também com 6 itens, e reconocimiento (ou reconhecimento), com quatro itens. Em sua versão validada, os autores apontam que o coeficiente do Alpha de Cronbach geral da ferramenta é $\alpha=0.608$. Já no fator 1 (ajuste), tem-se $\alpha=0.909$; no fator 2 (conflito) tem-se $\alpha=0.891$ e no fator 3 (reconhecimento), $\alpha=0.901$. Portanto, todos os alfas encontram-se dentro dos parâmetros estatísticos indicados por Cronbach (1951).

Cabe explicitar que a APO foi aplicada, assim como sua versão original, por meio de uma escala de 7 pontos do tipo Likert, variando de 0 - "Discordo totalmente", passando pelos 1 - "Discordo muito", 2 "Discordo pouco", 3 - "Não concordo nem discordo", 4 - "Concordo pouco", 5 - "Concordo muito", até o 6 - "Concordo totalmente".

Para a avaliação da satisfação no trabalho, levou-se em consideração, inicialmente, a utilização do instrumento Job Satisfaction in the Public Sector (JSPS), de Wright e Davis (2003), uma vez que essa ferramenta foi construída e validada especificamente para o setor público. Nele o construto é medido por meio de 31 itens, que se agrupam em oito fatores hipotéticos, sendo quatro deles referentes às características do trabalho (rotina, especificidade do trabalho, desenvolvimento de recursos humanos e feedback), e quatro referentes ao contexto do trabalho (conflito nas metas organizacionais, restrições processuais, especificidade das metas organizacionais e recomendações a terceiros). No entanto, observou-se que a escala não leva em consideração o fator hipotético "Satisfação com o Salário". Sendo esse um campo de constante conflito no serviço público - e não só nele -, historicamente objeto de diversos movimentos paredistas. Ainda, fundamentado por estudos seminais que visam a compreensão do construto satisfação no trabalho, tais como Locke (1976), Brief (1998) e George e Jones (1999), que afiançam que as variáveis que buscam avaliar a satisfação com o salário dentro do construto não devem ser desprezadas.

Assim, optou-se pela Escala de Satisfação no Trabalho (EST) de Siqueira (2008). Reforçando a escolha, de acordo com Traldi e Demo (2012), este é o instrumento mais utilizado no Brasil, sobretudo, em sua versão reduzida (escala composta de 15 itens). Cabe observar que a escala também já foi testada no serviço público brasileiro por Andrade (2019) com resultados consistentes.

Os 15 itens utilizados na EST $(\alpha=0,89)$, bem como sua escala na versão completa, apresentou 5 fatores com três variáveis cada:

- Fator 1 - satisfação com os colegas de trabalho $(\alpha=0,81)$;

- Fator 2 - satisfação com o salário $(\alpha=0,90)$;

- Fator 3 - satisfação com a chefia $(\alpha=0,84)$;

- Fator 4 - satisfação com a natureza do trabalho $(\alpha=0,77)$;

- Fator 5 - satisfação com as promoções $(\alpha=0,81)$.

A EST também foi aplicada, assim como sua versão original, numa escala do tipo Likert de 7 pontos, variando de 0 - "Totalmente insatisfeito", passando pelo 1 - "Muito insatisfeito", 2 - "Insatisfeito", 3 "Indiferente", 4 - "Satisfeito", 5 - "Muito satisfeito" até o 6 - "Totalmente satisfeito".

Para a avaliação do engajamento no trabalho, foi utilizada a Utrecht Work Engagement Scale (UWES), também em sua versão reduzida, de Schaufeli, Bakker e Salanova (2006). Essa versão, de acordo com os autores, possui alfa de Cronbach acima de 0,80 e foi desenvolvida a partir da versão original de 17 itens e três fatores. Sua redução resultou em nove itens e os mesmos três fatores hipotéticos, traduzidos como Vigor, Dedicação e Absorção. $\mathrm{O}$ estudo foi realizado em 10 países diferentes, com $\mathrm{n}=14.521$ indivíduos. Cabe ressaltar que o Brasil não fez parte dessa coleta e que as unidades de análise foram mistas, isto é, público e privado. Entretanto, na pesquisa de Andrade (2019), a escala demonstrou resultados estatísticos favoráveis à utilização no país e aplicação em servidores públicos.

Neste estudo, assim como na versão reduzida de Schaufeli et al. (2006), as respostas foram coletadas por meio de escala do tipo Likert de sete pontos, variando desde o 0 - "Nunca/Nenhuma vez", passando 
pelo 1 - "Quase nunca/Algumas vezes por ano", 2 - "Algumas vezes/Uma vez ou menos por mês", 3 "Regularmente/Algumas vezes por mês", 4 - "Bastantes vezes/Uma vez por semana", 5 - "Quase sempre/ Algumas vezes por semana" até o 6 - "Sempre/Todos os dias".

Por fim, todas as três ferramentas utilizadas passaram por adaptações na escrita, frente as originais, a fim de adequá-las à utilização da pesquisa na administração pública. Por exemplo, foram substituídos os termos "organização" por "instituição" e "colaborador" por "servidor", uma vez que estes concordam melhor com as unidades de análise e objeto e objetivos do estudo.

Para a coleta dos dados foi utilizado o programa online SurveyMonkey, ferramenta de distribuição e criação de questionários via web. Surveys eletrônicos, desde a popularização da internet, vêm mostrando-se atrativos para pesquisadores, pois permitem ampla e veloz divulgação de questionários substanciais e versatilidade, pois o respondente o faz de acordo com o seu tempo, baixo custo e retira o possível viés do entrevistador sob o entrevistado (Baulac, Bolden, \& Moscarola, 2000). Entretanto, ainda segundo os mesmos autores, um empecilho à utilização dessa modalidade é o baixo índice de respostas. Os questionários ficaram disponíveis entre 07 de março de 2018 e 18 de abril de 2018. Foram enviadas mensagens eletrônicas, uma vez que o pesquisador possui um banco de dados com o endereço de e-mail de servidores de diferentes regiões e esferas da administração pública, sendo assim encaminhado aos mesmos de forma nominal. Por fim, foi utilizada uma opção que não permitia que o respondente enviasse o questionário se ele não completasse todas as perguntas. Essa ação impossibilita a presença de dados faltantes, o que melhora a taxa de aproveitamento dos questionários respondidos.

Para a análise dos dados foi utilizada a técnica de modelagem de equações estruturais (MME), seguindo as recomendações de Hair, Hult, Ringle e Sarstedt (2016) para validação do modelo e demais testes das hipóteses. De maneira complementar, foi elaborada uma série de regressões hierárquicas para os testes de mediação utilizando a macro 1 do Process ${ }^{\circledR}$ proposto por Hayes (2017). Foram utilizados os softwares Smart PLS 2.0.M3 (Ringle, Wende, \& Will, 2005), e SPSS (v.21), respectivamente. Para estimação do tamanho mínimo da amostra foi utilizado o software da plataforma $G^{*}$ Power v.3.1.9.2. Os dados foram explorados inicialmente para a retirada de casos com dados faltantes ou extremos. $\mathrm{O}$ tamanho mínimo da amostra ficou bem abaixo do que foi coletado, um total de 902 questionários válidos. Também foi avaliada a normalidade dos dados para adequação ao método escolhido.

Para validação do modelo, observou-se inicialmente o fator de inflação da variância (VIF) de cada item menor que 5, seguida pela validade convergente e discriminante do modelo estrutural (Hair et al., 2016). Também foi avaliada a média de variância extraída (AVE). Foram avaliados para a mesma finalidade de robustez do modelo os indicadores de consistência interna (Alpha de Cronbach e Confiabilidade Composta), bem como as cargas fatoriais das variáveis manifestas de cada construto acima de 0,708 (o que permite explicar mais de $50 \%$ da variável que é mensurada) e com correlação maior nos seus respectivos construtos. Para aceitação das hipóteses e demais testes estatísticos foi considerado o valor de T de Student maior ou igual a 1,96 (p-valor menor ou igual a 5\%), por meio do teste de reamostragem com 5000 subamostras (bootstrapping). Para aceitação do efeito mediador da variável, também adotou-se o p-valor menor ou igual a 0,05 e a inexistência de zero dentro do intervalo de confiança do parâmetro testado para o beta da regressão. Por fim, a capacidade de explicação do modelo estrutural para a variável dependente, por meio do R., relevância preditiva (Q.) e tamanho do efeito (f.) complementam a validação do modelo e permitiram a comparação destes entre si, objeto deste estudo.

\section{ConfirmaçÃo dos Modelos}

As proposições passaram inicialmente por um processo de análise fatorial confirmatória em um modelo de base, buscando avaliar o ajuste geral aos dados. A Tabela 1 aponta os dados iniciais de validade convergente deste modelo. 
Diego César Terra de Andrade. Comprometimento Institucional: um modelo interpretativo Para o servi...

Tabela 1 - Validade convergente inicial

\begin{tabular}{|c|c|c|c|c|c|c|}
\hline Construto & AVE & $\begin{array}{l}\text { Confiabilicade } \\
\text { composta }\end{array}$ & $\mathrm{R}^{2}$ & $\begin{array}{l}\text { Alpha de } \\
\text { Cronbach }\end{array}$ & Comunalidade & Redundância \\
\hline 1.Absorção & 0,846 & 0,917 & 0,870 & 0,819 & 0,846 & 0,732 \\
\hline 2.Ajuste & 0,716 & 0,926 & 0,828 & 0,900 & 0,716 & 0,592 \\
\hline 3. Chefia & 0,872 & 0,953 & 0,542 & 0,926 & 0,872 & 0,473 \\
\hline $\begin{array}{l}\text { 4. Colegas de } \\
\text { trabalho }\end{array}$ & 0,756 & 0,903 & 0,515 & 0,839 & 0,756 & 0,389 \\
\hline 5.Conflito & 0,583 & 0,893 & 0,689 & 0,857 & 0,583 & 0,397 \\
\hline 6.Dedicação & 0,873 & 0,954 & 0,943 & 0,927 & 0,873 & 0,823 \\
\hline $\begin{array}{l}\text { 7. Natureza do } \\
\text { trabalho }\end{array}$ & 0,713 & 0,882 & 0,615 & 0,799 & 0,713 & 0,439 \\
\hline 8. Promoções & 0,797 & 0,922 & 0,643 & 0,872 & 0,797 & 0,513 \\
\hline 9. Reconhecimento & 0,666 & 0,856 & 0,364 & 0,747 & 0,666 & 0,242 \\
\hline 10. Salário & 0,877 & 0,955 & 0,447 & 0,930 & 0,877 & 0,392 \\
\hline 11.Vigor & 0,851 & 0,945 & 0,912 & 0,912 & 0,851 & 0,775 \\
\hline
\end{tabular}

Fonte: resultados da pesquisa.

Observa-se que os indicadores de AVE, R. e confiabilidade interna (Alpha de Cronbach e Confiabilidade composta) ficaram acima dos valores padrão, conforme sugeridos por Hair et al. (2016). A Tabela 2 apresenta, de maneira complementar, que a validade discriminante também está indicada e é consistente.

Tabela 2 - Validade discriminante

\begin{tabular}{|c|c|c|c|c|c|c|c|c|c|c|c|}
\hline Construto & 1 & 2 & 3 & 4 & 5 & 6 & 7 & 8 & 9 & 10 & 11 \\
\hline 1.Absorção & 0,920 & & & & & & & & & & \\
\hline 2.Ajuste & 0,436 & 0,846 & & & & & & & & & \\
\hline 3. Chefia & 0,337 & 0,415 & 0,934 & & & & & & & & \\
\hline $\begin{array}{l}\text { 4. Colegas de } \\
\text { trabalho }\end{array}$ & 0,348 & 0,414 & 0,514 & 0,870 & & & & & & & \\
\hline 5. Conflito & $-0,368$ & $-0,583$ & $-0,367$ & $-0,382$ & 0,764 & & & & & & \\
\hline 6.Dedicação & 0,883 & 0,501 & 0,378 & 0,390 & $-0,414$ & 0,935 & & & & & \\
\hline $\begin{array}{l}\text { 7. Natureza do } \\
\text { trabalho }\end{array}$ & 0,622 & 0,484 & 0,466 & 0,481 & $-0,421$ & 0,686 & 0,845 & & & & \\
\hline 8. Promoções & 0,408 & 0,503 & 0,459 & 0,408 & $-0,344$ & 0,486 & 0,528 & 0,893 & & & \\
\hline 9. Reconhecimento & 0,213 & 0,489 & 0,342 & 0,419 & $-0,269$ & 0,257 & 0,278 & 0,328 & 0,816 & & \\
\hline 10. Salário & 0,201 & 0,263 & 0,254 & 0,293 & $-0,178$ & 0,269 & 0,434 & 0,548 & 0,174 & 0,936 & \\
\hline 11.Vigor & 0,829 & 0,480 & 0,370 & 0,425 & $-0,402$ & 0,882 & 0,643 & 0,446 & 0,284 & 0,271 & 0,922 \\
\hline
\end{tabular}

Fonte: resultados da pesquisa.

Ainda para estabelecer a validade discriminante, na Tabela 3 os crossloadings podem ser analisados. O que se observa é que os itens carregaram mais em seus respectivos construtos do que nos demais. Pode-se, ainda, avaliar que os construtos se discriminaram, pois a raiz quadrada da AVE (indicadores em negrito), ficaram acima do valor de correlação do construto com os demais construtos do modelo. 
Tabela 3 - Crossloading do modelo geral

\begin{tabular}{|c|c|c|c|c|c|c|c|c|c|c|c|}
\hline$m$ & & & & 7 & 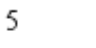 & $\sigma$ & & & & & \\
\hline & 31 & 52 & 69 & & 38 & 59 & & & & & \\
\hline & 09 & 33 & 44 & 59 & & 49 & 02 & 33 & 52 & 25 & 82 \\
\hline & 387 & & & & & & & & & & \\
\hline & 369 & & & & & $\$ 33$ & & & & & \\
\hline & 353 & 43 & 361 & 315 & & 393 & 99 & 393 & 40 & 07 & 390 \\
\hline & 8 & & & & & 05 & & & & & \\
\hline & 882 & 9 & 1 & 0 & & 49 & 04 & 42 & 48 & & 14 \\
\hline & 329 & 0 & 53 & & & & & & & & \\
\hline & 4 & & 52 & & & 50 & & & & & \\
\hline & 301 & 57 & 895 & 86 & & 339 & 19 & 24 & 39 & 09 & 318 \\
\hline & 281 & 9 & 385 & 55 & & 332 & & & & & \\
\hline & 311 & & 8 & & & 32 & & & & & \\
\hline & 314 & 66 & 82 & 90 & & 0,351 & & 52 & 82 & & 84 \\
\hline & 308 & 105 & $-0,288$ & & & 39 & & & & & \\
\hline & 14 & & 98 & & 1 & 85 & & & & & - \\
\hline & 75 & & $-0,229$ & 29 & 992 & $-0,286$ & & & & & \\
\hline & & & 27 & & & -0 & & & & - & \\
\hline & 99 & -0 , & $-0,224$ & 55 & & $-0,255$ & 79 & & & & - \\
\hline & . & - & -0 , & & & & & & & & 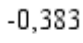 \\
\hline & 9 & & $0=$ & & & & & & & & \\
\hline & 29 & 38 & 367 & & & 0,950 & 33 & 79 & 39 & 44 & 27 \\
\hline & 9 & & & & & & & & & & \\
\hline & 355 & & 401 & & & 39 & & & & & \\
\hline & 57 & & & & & & & & & & \\
\hline & & & & & & & & & & & \\
\hline & 89 & 2 & 1 & 19 & 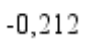 & 26 & 7 & 99 & 46 & 35 & 53 \\
\hline & & & & & & & & & & & \\
\hline & 155 & & & & & 39 & & & & & \\
\hline & 80 & 2 & 20 & & & 47 & 13 & & 3 & 7 & 50 \\
\hline & & & & & & & & & & & \\
\hline & 0,193 & 33 & 89 & 09 & 88 & 52 & 20 & 10 & 76 & 50 & 51 \\
\hline & & & & & & & & & & & \\
\hline & 0,472 & & & & & & & 0,451 & 61 & & 500 \\
\hline & 0,499 & & 0,430 & 85 & & 51 & 1 & 33 & 01 & 66 & 00 \\
\hline & & & & & & & & & & 1 & 940 \\
\hline & 0,747 & & 0,304 & & & & & 77 & 252 & 222 & 927 \\
\hline & 0,803 & 0,476 & 0,354 & 0,389 & $-0,372$ & 0,833 & 0,577 & 0,419 & 0,264 & 0,235 & 0,900 \\
\hline
\end{tabular}

Fonte: resultados da pesquisa.

A Tabela 4 apresenta a confirmação de todas as hipóteses levantadas no estudo. Tomados em conjunto, esses indicadores sustentam que o modelo estrutural de base alcançou um bom ajuste aos dados levantados, permitindo que se possa avaliar novos testes, como a comparação entre os três modelos propostos, bem como realizar testes adicionais de mediação, conforme o modelo avaliado. 
Tabela 4 - Teste de hipóteses

\begin{tabular}{|c|c|c|c|c|c|c|c|}
\hline Hipótese & Relaçäo estrutural & $\begin{array}{l}\text { Coeficiente } \\
\text { original }\end{array}$ & $\begin{array}{l}\text { Média das } 5000 \\
\text { subamostras }\end{array}$ & Erro padräo & Teste t & $\mathrm{p}$-valor & Status \\
\hline $\mathrm{H} 1$ & $\begin{array}{l}\text { ARTICULAÇÄO DE VALORES à SATISFAÇÄO } \\
\text { NO TRABALHO }\end{array}$ & 0,604 & 0,604 & 0,024 & 25,462 & 0,001 & Suportada \\
\hline $\mathrm{H}_{2}$ & $\begin{array}{l}\text { SATISFAC̆ÄO NO TRABALHO à ENGAJAMENTO } \\
\text { NO TRABALHO }\end{array}$ & 0,453 & 0,452 & 0,035 & 12,855 & 0,001 & Suportada \\
\hline H3 & $\begin{array}{l}\text { ARTICULAÇÄO DE VALORES à ENGAJAMENTO } \\
\text { NO TRABALHO }\end{array}$ & 0,245 & 0,247 & 0,038 & 6,494 & 0,001 & Suportada \\
\hline - & ARTICULAÇÄO DE VALORES à Ajuste & 0,910 & 0,910 & 0,007 & 139,453 & 0,001 & - \\
\hline - & ARTICULAÇÄO DE VALORES à Conflito & $-0,830$ & $-0,830$ & 0,015 & 55,712 & 0,001 & - \\
\hline - & ARTICULAÇÄO DE VALORES à Reconhecimento & 0,603 & 0,603 & 0,033 & 18,425 & 0,001 & - \\
\hline- & ENGAJAMENTO NO TRABALHO à Absorçäo & 0,933 & 0,933 & 0,006 & 158,134 & 0,001 & - \\
\hline - & ENGAJAMENTO NO TRABALHO à Dedicação & 0,971 & 0,971 & 0,003 & 381,113 & 0,001 & - \\
\hline - & ENGAJAMENTO NO TRABALHO à Vigor & 0,955 & 0,955 & 0,004 & 243,717 & 0,001 & - \\
\hline - & SATISFAÇÄO NO TRABALHO à Chefia & 0,736 & 0,737 & 0,021 & 35,215 & 0,001 & - \\
\hline - & $\begin{array}{l}\text { SATISFAÇÄO NO TRABALHO à Colegas de } \\
\text { trabalho }\end{array}$ & 0,718 & 0,718 & 0,022 & 33,177 & 0,001 & - \\
\hline- & $\begin{array}{l}\text { SATISFAÇÄO NO TRABALHO à Natureza do } \\
\text { trabalho }\end{array}$ & 0,784 & 0,785 & 0,018 & 42,682 & 0,001 & - \\
\hline - & SATISFAÇÄO NO TRABALHO à Promoçőes & 0,802 & 0,802 & 0,016 & 51,904 & 0,001 & - \\
\hline - & SATISFAÇÄO NO TRABALHO à Salário & 0,669 & 0,668 & 0,029 & 23,245 & 0,001 & - \\
\hline
\end{tabular}

Fonte: resultados da pesquisa.

Todas as 3 hipóteses foram confirmadas no modelo inicial e em todos os demais modelos também, o que confirma equação referente ao modelo integrativo anteriormente proposta.

No modelo 1, quanto maior a Articulação dos Valores Organizacionais e Pessoais, maior será a Satisfação no Trabalho $(\Gamma=0,604, \mathrm{t}=25,462, \mathrm{p}<0,001)$. A Satisfação no Trabalho também eleva significativamente o Engajamento no Trabalho $(\Gamma=0,453, t=12,855, \mathrm{p}<0,001)$ e, , por fim, a dos Valores Organizacionais e Pessoais leva a um maior Engajamento no Trabalho $(\Gamma=0,245, \mathrm{t}=6,494, \mathrm{p}<0,001)$.

No modelo 2, quanto maior a Satisfação no Trabalho, maior será o Engajamento no Trabalho $(\Gamma=0,601$, $\mathrm{p}<0,001)$, e quanto maior o Engajamento no Trabalho, maior será a Articulação dos Valores Organizacionais e Pessoais $(\Gamma=0,243, \mathrm{p}<0,001)$.

No modelo 3, quanto maior o Engajamento no Trabalho, maior será a Articulação dos Valores Organizacionais e Pessoais $(\Gamma=0,519, \mathrm{p}<0,001)$, e quanto maior a Articulação dos Valores Organizacionais e Pessoais, maior será a Satisfação no Trabalho $(\Gamma=0,399, \mathrm{p}<0,001)$. 
Após o ajuste do modelo estrutural, passou-se à comparação dos modelos 1, 2 e 3. Como parâmetros principais, os indicadores de explicação da variância total da variável dependente, R. e Q., foram escolhidos para esta avaliação, bem como o valor preditivo das variáveis do modelo (f.). Além disso, testes adicionais de mediação permitiram explorar aspectos teóricos complementares.

\section{Comparação dos Modelos}

Para Hair et al. (2016), o melhor ajuste de um modelo estrutural se dá pela sua capacidade de explicação da variável dependente. Uma busca por modelos alternativos, na qual se faz uma análise comparativa da qualidade de ajuste de dois ou mais modelos teóricos previamente especificados, permite que se construa uma melhor explicação teórica para o fenômeno investigado. Nesse sentido, as relações de caminho entre as variáveis, os indicadores gama $(\Gamma)$, de todas as relações bivariadas entre um construto e outro possuem valores distintos, independente do modelo. A Tabela 5 permite avaliar os indicadores de ajuste de cada modelo.

Tabela 5 - Comparação entre os modelos

\begin{tabular}{|c|c|c|c|c|c|c|c|}
\hline \multirow[b]{2}{*}{ Construtos } & \multicolumn{2}{|c|}{ Modelo 1} & \multicolumn{2}{|l|}{ | Modelo 2} & \multicolumn{3}{|l|}{ Modelo 3} \\
\hline & $\mathrm{R}^{2}$ & $\mathrm{Q}^{2}$ & $\mathrm{R}^{2}$ & $Q^{2}$ & $\mathrm{R}^{2}$ & $Q^{2}$ & $\mathrm{f}^{2}$ \\
\hline $\begin{array}{l}\text { ARTICULAÇÄO DE } \\
\text { VALORES }\end{array}$ & - & - & $40,20 \%$ & 0,171 & $26,90 \%$ & 0,114 & 0,363 \\
\hline Ajuste & 0,828 & 0,598 & 0,828 & 0,597 & 0,828 & 0,598 & - \\
\hline Conflito & 0,689 & 0,402 & 0,689 & 0,402 & 0,689 & 0,402 & - \\
\hline Reconhecimento & 0,364 & 0,250 & 0,364 & 0,252 & 0,364 & 0,250 & - \\
\hline $\begin{array}{l}\text { SATISFAÇÄO NO } \\
\text { TRABALHO }\end{array}$ & $36,50 \%$ & 0,154 & - & - & $47,50 \%$ & 0,207 & 0,367 \\
\hline Colegas de trabalho & 0,515 & 0,401 & 0,515 & 0,401 & 0,515 & 0,400 & - \\
\hline Salário & 0,447 & 0,396 & 0,447 & 0,396 & 0,447 & 0,401 & - \\
\hline Chefia & 0,542 & 0,493 & 0,542 & 0,493 & 0,542 & 0,493 & - \\
\hline Natureza do trabalho & 0,615 & 0,449 & 0,615 & 0,449 & 0,615 & 0,446 & - \\
\hline Promoções & 0,643 & 0,523 & 0,643 & 0,523 & 0,643 & 0,524 & - \\
\hline $\begin{array}{l}\text { ENGAJAMENTO NO } \\
\text { TRABALHO }\end{array}$ & $39,90 \%$ & 0,316 & $36,10 \%$ & 0,289 & - & - & 0,707 \\
\hline ז r:-... & nnon & ח & nnin & ก 701 & nnon & יחר ח & \\
\hline
\end{tabular}

Dentre os modelos testados, aquele que maximiza a explicação da variável dependente é o modelo 3 ( R. $=47,5 \%, Q .=0,207)$, em que a Satisfação no Trabalho é explicada como consequente da Articulação dos Valores Organizacionais e Pessoais $(\Gamma=0,399, \mathrm{p}<0,001)$ e do Engajamento no Trabalho $(\Gamma=0,392$, $\mathrm{p}<0,001)$. Nesse cenário, o Comprometimento Institucional se dá pela influência da Articulação dos Valores Organizacionais e Pessoais e do Engajamento no Trabalho sobre a Satisfação no Trabalho.

O modelo de base aponta que o Engajamento no Trabalho é o construto que possui um papel influenciador principal no modelo $(f .=0,707)$, e um equilíbrio de peso entre a Satisfação no Trabalho e a Articulação dos Valores Organizacionais e Pessoais ( $f_{.}=0,0,367$ e 0,363 , respectivamente).

Vale analisar que nos modelos testados existe sempre um papel mediador intermediário. No modelo 1, esse papel mediador foi exercido pela Satisfação no Trabalho para a relação entre Articulação dos Valores Organizacionais e Pessoais e Engajamento no Trabalho, e confirmado estatisticamente (efeito=0,223, IC $[0,145 ; 0,312])$. Esse aspecto enfatiza ainda mais um papel central da Satisfação no Trabalho sob o Comprometimento Institucional.

Quando se avalia a mediação do Engajamento no Trabalho para a relação entre a Satisfação no Trabalho como antecedente e a Articulação dos Valores Organizacionais e Pessoais como variável dependente, não se confirma tal papel condicional (efeito=0,002, IC[- 0,031;0,033]). 
Por fim, o proposto modelo 3 resulta como o ideal para interpretar o Comprometimento Institucional no serviço público brasileiro, a Articulação dos Valores Organizacionais e Valores Pessoais é que medeia a relação entre o Engajamento no Trabalho e a Satisfação no Trabalho (efeito=0,011, IC [ 0,004;0,021]).

A representação gráfica do modelo estrutural final pode ser observado na Figura 2.

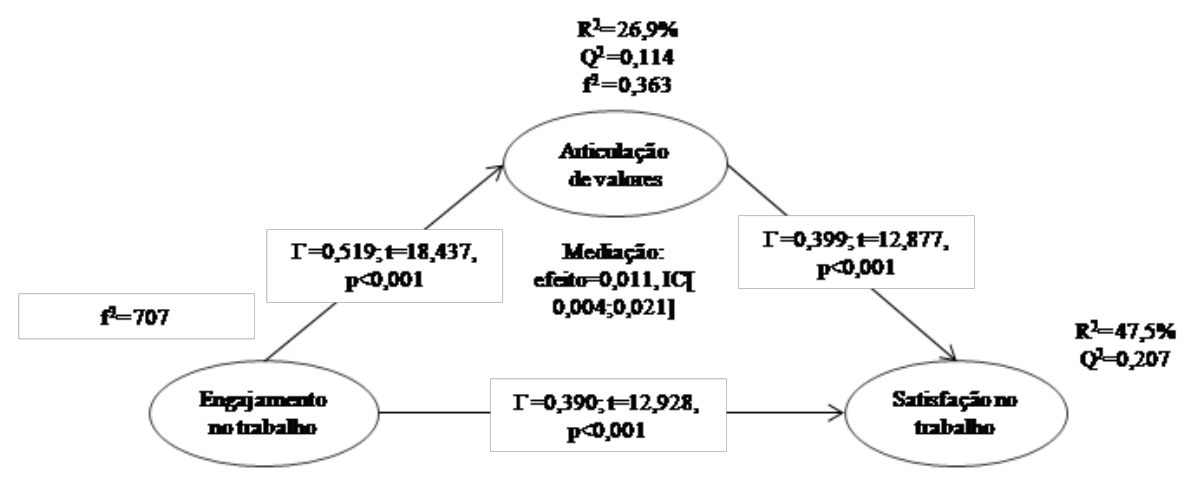

Figura 2 - Modelo estrutural final

Fonte: resultados da pesquisa.

\section{Discussão e Considerações Finais}

O modelo final aqui posto (Figura 2), passou por uma validação rigorosa na análise confirmatória, e bastante detalhada de suas variações. A capacidade preditiva do modelo explica que a Satisfação no Trabalho é a consequente ideal para interpretar o Comprometimento Institucional ( $\mathrm{R} .=47,5 \%$ e Q. $=0,207)$. O modelo traz ainda a contribuição teórica do papel mediador da Articulação dos Valores Organizacionais e Valores Pessoais (efeito=0,011, IC[ 0,004;0,021]), com o Engajamento no Trabalho como antecedente principal $(\mathrm{f} .=0,707)$, em uma amostra bastante representativa de funcionários públicos $(\mathrm{n}=902)$. Nesse sentido, o trabalho cumpre seu objetivo e ainda avança em propor uma discussão sobre variáveis mediadoras do processo de comprometimento institucional de funcionários públicos brasileiros.

Quando se analisa na literatura outros resultados, que apontam as consequências da Satisfação no Trabalho, Cunha et al. (2016) são categóricos ao afirmarem que duas questões básicas são necessárias nessa discussão. A primeira diz respeito à relação entre a satisfação com o trabalho e a satisfação com a vida. Nesse sentido, Judge e Klinger (2007) sugerem que a satisfação com trabalho pode influenciar a satisfação com a vida, embora a relação inversa também possa ocorrer. Portanto, trata-se de uma relação bidirecional.

Andrade (2019) expõem que a formação dos valores organizacionais é fruto do grupo que ali trabalha. No entanto, dentro de uma dinâmica viva, um segue influenciando o outro (Borges, Argolo, Pereira, Machado, \& Silva, 2002), ou seja, os valores organizacionais moldam os valores pessoais, ao mesmo tempo que os valores pessoais moldam os valores organizacionais - trata-se, também, de uma relação bidirecional. O que se conclui é que as relações de influência bidirecionais têm surgido como mediadoras do construto Satisfação no Trabalho, corroborando o encontrado nesta pesquisa.

Essas relações de influência bidirecionais, do ponto de vista de uma abordagem funcionalista, podem ser de complexa intervenção. Por exemplo, uma política de gestão de pessoas, para ser efetiva ao buscar aumentar os níveis de satisfação do trabalhador, teria que abarcar questões de ordem individuais, da vida e do trabalho, o que, muitas vezes, extrapola o alcance da organização ou instituição.

As buscas por efetividade nas políticas de gestão de pessoas trazem a segunda questão básica dessa discussão. Cunha et al. (2016) advogam que a satisfação com o trabalho tem implicações nos comportamentos dos membros da organização, a saber: "são mais assíduas e pontuais, abandonam menos a organização e adotam mais o comportamento de cidadania organizacional” (p. 160). No mesmo sentido, poderia se pensar que o engajamento no trabalho é um consequente da satisfação no trabalho. De fato, há um crescente corpo de 
evidências que relacionam o engajamento no trabalho como resultado organizacional, como por exemplo Harter, Schmidt e Hayes, (2002), Laschinger e Finegan (2005), Laschinger e Leiter (2006), Salanova, Agut e Peiro (2005) e Schaufeli e Bakker (2004).

Entretanto, o entendimento se o construto Engajamento no Trabalho é um antecedente ou consequente no contexto organizacional não constitui solução pacificada. Na busca por essa resposta, tem-se, por exemplo, o trabalho de Saks (2006). O autor a partir da proposição de um modelo em que apresenta como hipótese dois núcleos, características do trabalho e características da organização, busca essa resposta em amostra com $\mathrm{n}=102$. Destarte, os resultados obtidos, após regressão múltipla, apontaram que o construto Satisfação no Trabalho é um consequente do Engajamento no Trabalho $(\mathrm{R} .=0.37, .<0.001)$. Corroborando os resultados obtidos nessa amostra com servidores públicos brasileiros.

Conclusivamente, o modelo 3, aqui apontado como o mais robusto, e\# um esboço da realidade. Ele não se limita a descrever, ele busca explicitar as relações estruturais entre os elementos do fenômeno estudado. Contudo, essa é uma das formas de se interpretar o comportamento organizacional e, particularmente, para elaboração de instrumentos para a sua avaliação, uma vez que a realidade axiológica da organização pode ser representada por meio dessa representação gráfica, mas a adequação do modelo para representar o fenômeno estudado deve ser verificada por meio de estratégias e procedimentos metodológicos apropriados. No entanto, o comportamento organizacional, como qualquer outra realidade, pode ser abordado a partir de vários modelos, e estes constituem diversas aproximações da mesma realidade, que é deveras complexa, multifacetada e dinâmica.

Como limites dessa pesquisa e sugestão de estudos futuros, a possibilidade de identificação de mais processos condicionais pode apontar novos caminhos teóricos para pesquisadores do tema. A agenda em que se teste novas variáveis mediadoras para que possa aumentar o conhecimento sobre o fenômeno, pode trazer outras contribuições acerca dos fenômenos do comportamento organizacional, sobretudo no setor público brasileiro que, por suas peculiaridades, apresenta realidade tão diversa da encontrada no setor privado.

\section{REFERÊNCIAS}

Aaker, D. A., Kumar, V., \& Day, G. S. (2001). Pesquisa de marketing. São Paulo: Atlas.

Allen, N. J., \& Meyer, J. P. (1996). Affective, continuance, and normative commitment to the organization: An examination of construct validity. Journal of vocational behavior, 49(3), 252-276.

ANDRADE, D. C. T. (2019). Engajamento no Trabalho no Serviço Público: um modelo multicultural. Revista de Administração Contemporânea, 23(5), 639-660.

Andrade, D. C. T., \& Ramos, H. R. (2018). Proposição de um Modelo de Engajamento no Trabalho no Contexto do Serviço Público Brasileiro e Português. In: XLII Encontro da Associação Nacional de Pós-Graduação e Pesquisa em Administração. Curitiba. Anais.

Babbie, E. (1996). The practice of social research. Belmont: Wadsworth Publishing Company.

Bakker, A. B., Schaufeli, W. B., Leiter, M. P., \& Taris, T. W. (2008). Work engagement: an emerging concept in occupational health psychology. Work \& Stress, 22(3), 187-200.

Baulac, Y., Bolden, R., \& Moscarola, J. (2000). Interactive Research: How Internet technology could revolutionise the survey and analysis process. In Londres: Association for Survey Computing Conference on Survey Research on the Internet, Imperial College (Vol. 28).

Borges, L. O., Argolo, J. C. T., Pereira, A. L. D. S., Machado, E. A. P., \& Silva, W. S. D. (2002). A síndrome de burnout e os valores organizacionais: um estudo comparativo em hospitais universitários. Psicologia: reflexão e crítica, 15(1), 189-200.

Brief, A. P. (1998). Attitudes in and around organizations (Vol. 9). Thousand Oaks, CA: Sage.

Brujne, P. D., Herman, J., \& Schoutheete, M. D. (1991). Dinâmica de pesquisa em ciências sociais. Rio de Janeiro: Francisco Alves. 
Burrel, G., Morgan, G. (1982). Sociological Paradigms and Organizational Analysis. London: Heineman.

Canova, K. R., \& Porto, J. B. (2010). O impacto dos valores organizacionais no estresse ocupacional: um estudo com professores de ensino médio. Revista de Administração Mackenzie, 11(5), 4-31.

Cooper-Hakim, A., \& Viswesvaran, C. (2005). The construct of work commitment: testing an integrative framework. Psychological bulletin, 131(2), 241-259.

Creswell, J.W. (2014). Research Design: Qualitative, Quantitative, and Mixed Methods Approaches. 4th ed. SAGE Publications: Thousand Oaks, California.

Cronbach, L. J. (1951). Coefficient alpha and the internal structure of tests. Psychometrika, 16(3), 297-334.

Cunha, M. P., Rego, A., Cunha, R. C., \& Cabral-Cardoso, C. (2016). Manual de comportamento organizacional e gestão ( $8^{a}$ ed.). Lisboa: Editora RH.

George, J. M., \& Jones, G. R. (1999). Understanding and managing organizational behavior (2. ed.). Reading, MA: Addison-Wesley.

Hair, J. F. Jr., Hult, G. T. M., Ringle, C., \& Sarstedt, M. (2016). A primer on partial least squares structural equation modeling (PLS-SEM). Los Angeles: Sage publications.

Hair, J. F. Junior, Anderson, R. E., Tatham, R. L., \& Black, W. C. (2005). Análise multivariada de dados. Porto Alegre: Bookman.

Harter, J.K., Schmidt, F. L., \& Hayes, T. L. (2002), "Business-unit level relationship between employee satisfaction, employee engagement, and business outcomes: a meta-analysis", Journal of Applied Psychology, 87(1), 268-79.

Hayes, A. F. (2017). Introduction to mediation, moderation, and conditional process analysis: A regression-based approach. New York: Guilford Publications.

Jaramillo, F., Mulki, J. P., \& Marshall, G. W. (2005). A meta-analysis of the relationship between organizational commitment and salesperson job performance: 25 years of research. Journal of Business Research, 58(6), 705-714.

Jayaratne, S. (1993). The antecedents, consequences, and correlates of job satisfaction. In R. T. Golembiewski (Ed.), Handbook of organizational behavior, (p.p 111, 140). New York: Marcel Dekker.

Judge, T. A., \& Klinger, R. (2007). Job satisfaction: Subjective well-being at work. In M. Eid \& R. Larsen (Eds.), The science of subjective well-being (pp. 393-413). New York: Guilford Publications.

Kluckhohn, C. \& Strodtbeck, F. L. (1961). Variations in value orientations. Evanston, III.: Row, Peterson.

Locke, E. A. (1976). The nature and causes of job satisfaction. Handbook of industrial and organizational psychology. (pp. 1297-1349). Chicago: Rand McNally.

Malhotra, N. K. (2006). Pesquisa de marketing: uma orientação aplicada. 4. ed. Porto Alegre: Bookman.

Meleiro, A. R. \& Siqueira, M. M. M. (2005). Os impactos do suporte do supervisor e de estilos de liderança sobre bem-estar no trabalho. Associação Nacional de Pós-Graduação e Pesquisa em Administração (Org.), Anais do Encontro Nacional da Associação Nacional de Pós-Graduação e Pesquisa em Administração. Brasília, DF, Brasil, 29.

Meyer, J. P., Stanley, D. J., Herscovitch, L., \& Topolnytsky, L. (2002). Affective, continuance, and normative commitment to the organization: A meta-analysis of antecedents, correlates, and consequences. Journal of vocational behavior, 61(1), 20-52.

Morrow, P. C. (2011). Managing organizational commitment: Insights from longitudinal research. Journal of vocational behavior, 79(1), 18-35.

Mowday, R. T., Porter, L. W. E, \& Steers, R. M. Employee-Organization linkages - the psychology of commitment, absenteism and turnover. New York. Academic Press. 1982.

Nelson, R. E. (2011). Adversity, Organizational culture and executive turnover in a Brazilian manufacturer. Organization Studies, 32(3), 407-425.

Oliveira, A. D. F., \& Tamayo, A. (2004). Inventário de perfis de valores organizacionais. Revista de Administração, 39(2), 129-140.

Padovam, V. A. R. (2005). Antecedentes de bem-estar no trabalho: percepções de justiça e suportes. 119 f. Dissertação (Mestrado em Psicologia da Saúde) - Faculdade de Psicologia e Fonoaudiologia, Universidade Metodista de São Paulo, 2005. 
Ramos, V., Jordão, F., \& Morais, T. (2013). Desarrollo y validación del Inventario para medir la articulación entre la persona y la organización (Inventario APO). Revista Evaluar, 12(1), 60-82.

Riketta, M. (2005). Organizational identification: A meta-analysis. Journal of vocational behavior, 66(2), 358-384.

Ringle, C. M., Wende, S., \& Will, A. (2005). SmartPLS 2.0. M3. Hamburg: SmartPLS. Retrieved from Available at: http://www. smartpls. com.

Rokeach, M. (1973). The nature of human values (Vol. 438). New York: Free press.

Saks, A. M. (2006). Antecedents and consequences of employee engagement. Journal of managerial psychology, 21(7), 600-619.

Salanova, M., Agut, S., \& Peiró, J. M. (2005). Linking organizational resources and work engagement to employee performance and customer loyalty: the mediation of service climate. Journal of applied Psychology, 90(6), 1217.

Schaufeli, W. B., Bakker, A. B., \& Salanova, M. (2006). The measurement of work engagement with a short questionnaire a cross-national study. Educational and psychological Measurement, 66(4), 701-716.

Schaufeli, W. B., Salanova, M., González-Romá, V., \& Bakker, A. B. (2002). The measurement of engagement and burnout: a two sample confirmatory factor analytic approach. Journal of happiness studies, 3(1), 71-92.

Schneider, B. (1987). The people make the place. Personnel psychology, 40(3), 437-453.

Schneider, B., Goldstein, H. W., \& Smith, D. B. (1995). The ASA framework: an update. Personnel psychology, 48(4), 747-773.

Schwartz, S. H. (1994). Are There Universal Aspects in the Structure and Contents of Human Values?. Journal of Social Issues, 50(4), 19-45.

Schwartz, S. H. (2005). Validade e aplicabilidade da teoria de valores. Em: Tamayo, A.; Porto, J. B. Valores e comportamentos nas organizações (pp. 56-95). Petrópolis: Vozes.

Siqueira, M. M. M. (1995). Antecedentes de comportamentos de cidadania organizacional: análise de um modelo póscognitivo. 265 f. Tese (Doutorado em Psicologia) - Instituto de Psicologia, Universidade de Brasília, Brasília, 1995.

Siqueira, M. M. M. (2003). Proposição e análise de um modelo para comportamentos de cidadania organizacional. Revista de Administração Contemporânea, 7(SPE), 165-184.

Siqueira, M. M. M. (2005). Esquema mental de reciprocidade e influências sobre afetividade no trabalho. Estudos de Psicologia, 10(1), 83-93

Siqueira, M. M. M. (2008). Satisfação no trabalho. Em Siqueira, M. M. M. (Org.). Medidas do comportamento organizacional: ferramentas de diagnóstico e de gestão (pp. 265-274). Porto Alegre: Artmed.

Staw, B. M. (1986). Organizational psychology and the pursuit of the happy/productive worker. California Management Review, 28(4), 40-53.

Tamayo, A. (1998). Valores organizacionais: sua relação com satisfação no trabalho, cidadania organizacional e comprometimento afetivo. Revista de Administração, 33(3), 56-63.

Tamayo, A. (2007). Impactos dos valores da organização sobre o estresse ocupacional. RAC Eletrônico, 1(2), 20-33.

Torres, C. V., Porto, J. B., Vargas, L. M., \& Fischer, R. (2015). Uma meta-análise dos valores humanos básicos no Brasil: diferenças observadas no país. Revista Psicologia, 15(1), 89-102.

Traldi, M. T. F., \& Demo, G. (2012). Comprometimento, bem-estar e satisfação dos professores de administração de uma universidade federal. REAd-Revista Eletrônica de Administração, 18(2), 290-316.

Vroom, V.H. (1966). Organizational choice: A study of pre-and postdecision processes. Organizational behavior and buman performance, 1(2), 212-225.

Wright, B. E., \& Davis, B. S. (2003). Job satisfaction in the public sector the role of the work environment. The American Review of Public Administration, 33(1), 70-90.

Wright, B. E., \& Davis, B. S. (2003). Job satisfaction in the public sector the role of the work environment. The American Review of Public Administration, 33(1), 70-90. 